\title{
A Comparison of Muscle Activation during the Pull-up and Three Alternative Pulling Exercises
}

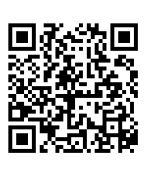

\author{
Jennifer K Hewit*, Daniel A Jaffe and Todd Crowder \\ Human Performance Laboratory, Department of Physical Education, USA
}

Submission: October 24, 2018; Published: November 02, 2018

*Corresponding author: Jennifer K Hewit, Human Performance Laboratory, Department of Physical Education, USA

\section{Introduction}

The pull-up is primarily an upper body exercise used to train the muscular endurance or muscular strength components of fitness. This challenging exercise requires the participant pull their entire body weight from a dead hang using only their upper body musculature. Many individuals struggle with being able to complete one repetition (rep) as well as increasing the maximum number of reps they are able to complete. As a result, training for improved pull-up performance can be quite frustrating as an individual is often not able to assess how close they are to being able to complete a pull-up until they successfully do so. Being able to train for the pull-up through similar modified or alternative exercises would assist with both progress tracking and assessment, as well as developing appropriate exercise-specific periodized programs Astrand, Rodahl, Dahl and Stromme [1], Bompa [2], MacIntosh, Gardiner and McComas [3].

Exercises that are often used in training programs to improve upper body pulling strength and muscular endurance include the seated lat-pulldown, assisted pull-up/chin-up machine, Baumgartner Modified Pull-up, Band/partner-assisted pull-ups, kipping pull-ups, suspended pull-ups, etc. Baumgartner and Gaunt [4] Halet, Mayhew, Murphy and Fanthorpe [5], Johnson, Lynch, Nash, Cygan and Mayhew [6]; Ronai \& Scibek [7], Snarr, Casey, Hallmark and Esco [8], Snarr, Hallmark, Casey and Esco [9]. While all of these exercises utilize the pulling motion, some are likely more similar to the traditional pull-up exercise than others. There appears to be three key features of interest when attempting to determine which of these alternative exercises might have the greatest transference to the traditional pullup. The first is the magnitude of muscle activation in the prime movers (i.e. latissimus dorsi, biceps brachii, trapezius, deltoid, erector spinae, rectus abdominis, etc. Dickie, Faulkner, Barnes and Lark [10], Snarr [9]. Activating the main muscles used in the alternative pulling exercises to a similar extent as that of the pullup will result in maximal transference from the training program and exercises to the exercise of interest; the body weight pull-up.

The second key feature of interest in the alternative exercise is pulling the body in the vertical direction, against the force of gravity Ronai and Scibek [4]. As the body and pulling motion becomes more horizontal, it is reasonable to assume that the muscle recruitment patterns will also be adjusted (e.g. increased rhomboid and middle trapezius activity). By keeping the motion in the vertical direction, the same muscle groups will likely be used. Therefore, exercises that do not maintain a vertically aligned body position throughout a vertical pulling motion will not be used as an alternative pulling exercise for this study (e.g. Baumgartner Modified Pull-up, suspended pull-ups, inverted rows, etc.). Finally, when designing a periodized training program, it is important to be able to track and adjust the load of the exercises to ensure that progress is being made and that plateaus can be minimized or avoided all together Astrand [1] Bompa [2], MacIntosh [3].

In order to do this, the amount of weight being moved during the alternative exercise must be known and easily increased (or decreased) as the training program progresses through macrocycles. While suspension exercises are able to increase and decrease the load of the exercise, it is more difficult to determine the exact load being lifted when using elastic resistance bands, partner assistance, or other suspension devices. Additionally, pull-ups performed utilizing excessive lower body motion (i.e. kipping) integrate a greater amount of momentum when performing the pulling action than that of the traditional pullup from a dead hang. As previous research has reported, the muscular demand of the upper body is significantly lower when performing kipping pull-ups when compared to that of traditional pull-ups Snarr [8]. Therefore, exercises of this nature will also not be used as alternative pulling exercises for this study (e.g. band/ partner-assisted pull-ups, feet suspended pull-ups, kipping pullups, etc.). Considering these three key factors for comparison of the alternative exercises with the pull-up, the exercises with the greatest transference potential appears to be the seated lat pulldown and assisted pull-up machine.

While the assisted pull-up requires the same body position of the traditional pull-up from the hips up, the seated position of the lat-pulldown may recruit the core stabilizing muscles (e.g. rectus abdominis, transverse abdominis, erector spinae, etc.) and hip flexors (e.g. iliopsoas and rectus femoris) to a lesser extent as a 
result of being supported in the seated position throughout the exercise. Performing the lat-pulldown from a kneeling position would create the neutral hip position that is more consistent with the traditional pull-up (and assisted pull-up) exercise. Research has recently begun to investigate the muscle activity of the latpulldown when varying the hand grip width and technique Dickie [10]; Lusk, Hale and Russell [11], Signorile, Zink and Szwed [12], Sperandei, Barros, Silveira-Junior and Oliveira [13]. However, to the knowledge of the authors of this study, the activation magnitudes of the prime movers during alternative pulling exercises compared to that of the BW pull-up has yet to be explored. Therefore, the purpose of this study is to determine if the magnitudes of muscle activation in the prime movers of one of the alternative pulling exercises (i.e. seated lat-pulldown, kneeling lat-pulldown and assisted pull-up machine) is more similar than the other two to the magnitudes of muscle activation in the traditional body weight pull-up. It was hypothesized that the seated lat-pulldown would have the weakest correlation and smallest shared variance with the pull-up due primarily to a decrease in activation of the rectus abdominis because of the seated position during the exercise.

\section{Methods}

Forty-one uninjured college-aged males $(\mathrm{n}=28)$ and females ( $n=13$ ) participated in this study (see Table 1 for subject characteristics). All procedures were approved by the United States Military Academy Human Research Protection Program before commencing the study. Prior to participation, an informed written consent was obtained from each subject. All testing took place in the USMA Human Performance Laboratory.

Table 1: Subject characteristics by group, represented by the Mean (SD).

\begin{tabular}{|c|c|c|}
\hline & $\mathbf{u 1 0}$ & $\mathbf{1 0 +}$ \\
\hline Males & 3 & 25 \\
\hline Females & 11 & 2 \\
\hline Age (years) & $20.9(3.8)$ & $22.6(4.8)$ \\
\hline Height (m) & $1.63(0.08)$ & $1.74(0.19)$ \\
\hline Weight $(\mathrm{kg})$ & $67.6(9.1)$ & $84.8(11.9)$ \\
\hline
\end{tabular}

\section{Procedures}
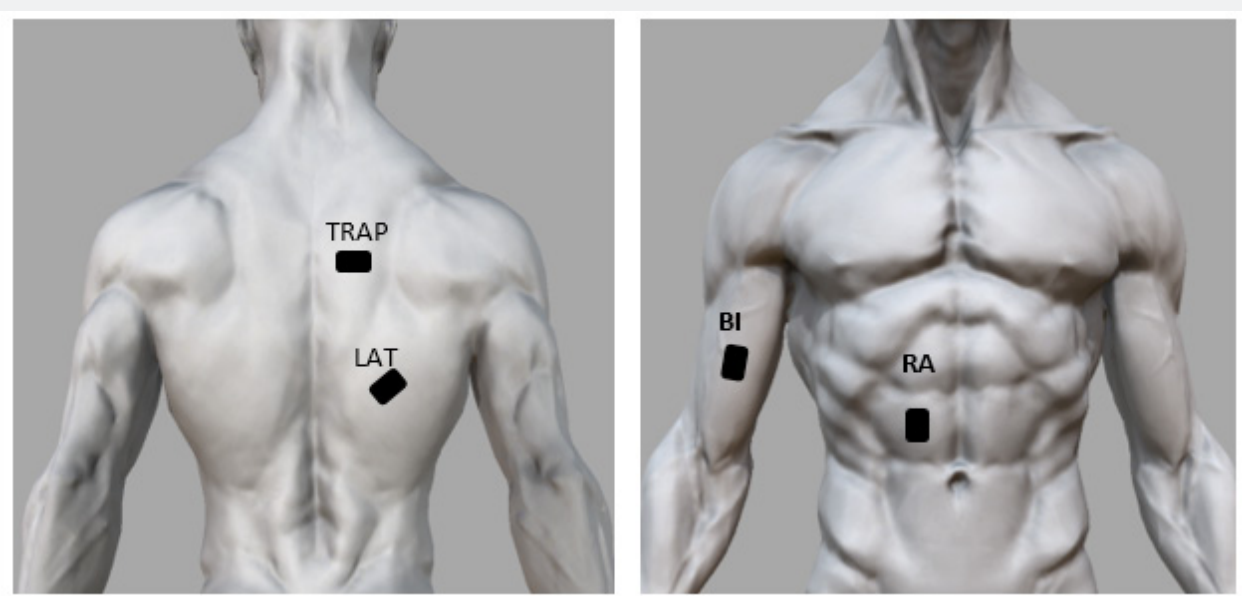

Figure 1: EMG placement for the four muscle groups of interest, where TRAP = middle fibers of trapezius, LAT = latissimus dorsi, $\mathrm{BI}=$ biceps brachii, and RA = rectus abdominis. Placement was standardized using the following measurements as described by Snarr [9]: TRAP $=50 \%$ of the distance between the spinous process of the thoracic vertebrae and the medial border of the spine of the scapula, at an angle perpendicular to the vertebral column; LAT $=4 \mathrm{~cm}$ below the inferior angle of the scapula, and $50 \%$ of the distance between the lateral border of the torso and the spinous process of the vertebrae, at an oblique angle towards the greater tubercle of the humerus; $\mathrm{BI}=$ centered over the flexed muscle belly, vertically aligned with the upper arm; and RA $=50 \%$ of the distance between the xyphoid process and naval, and $50 \%$ of the distance between the linea alba and ribs.

Upon arrival to the single testing session, the age, height, weight and max number of pull-ups performed in a single bout (self-reported) were recorded for each participant. Investigators then led the participants through a 5-minute standardized warmup which focused on shoulder and thoracic mobility. Wireless surface electromyography (EMG) electrodes (Delsys, Inc., Natick, MA, USA) were then placed on the right side of the body, centered over the muscle belly of the latissimus dorsi (LAT), trapezius (middle fibers) (TRAP), biceps brachii (BI), and rectus abdominis (RA) see in Figure 1. A 5-second maximum voluntary isometric contraction (MVIC) was recorded for each of the four muscles; a modified lat pulldown (i.e. supinated grip, shoulder width hand placement on bar, pulling down until the elbows are parallel to the shoulders) was used for the LAT and BI, a seated row (pulling back until the upper arms were in line with the torso) for the TRAP, and a resisted curl-up (arms placed across the chest, knees bent to $90^{\circ}$; the participant curls up approximately $30^{\circ}$ off the ground and is manually resisted at the shoulders) for the RA Konrad [14]. Following MVIC data collection, participants were allowed to familiarize themselves with the 4 exercises. No more than 3 repetitions of each exercises were taken by any of the participants.

Participants performed 5 repetitions of each of the 4 exercises; body weight (BW) pull-up (PU), seated lat-pulldown (sLP) at $80 \%$ BW, kneeling lat-pulldown (kLP) at $80 \%$ BW, and assisted pullup (aPU) at 20\% BW deficit see in Figures 2-5 Johnson [6]. Hand 
placement was standardized across subjects as $1.5 \mathrm{x}$ bi-acromial width Andersen, Fimland, Wiik, Skoglund and Saeterbakken [15]. The order of exercises was randomly assigned using a block randomization design. For example, Participant 1 would perform sLP, kLP, aPU and finally PU. Participant 2 would perform kLP, aPU,
PU, and finally, sLP. Participant 3 would perform aPU, PU, sLP, and finally kLP. Participant 4 would perform PU, sLP, kLP, and finally aPU. Participant 5 would perform the exercises in the same order as Participant 1. A recovery period of 5 minutes was given to subjects between each exercise.

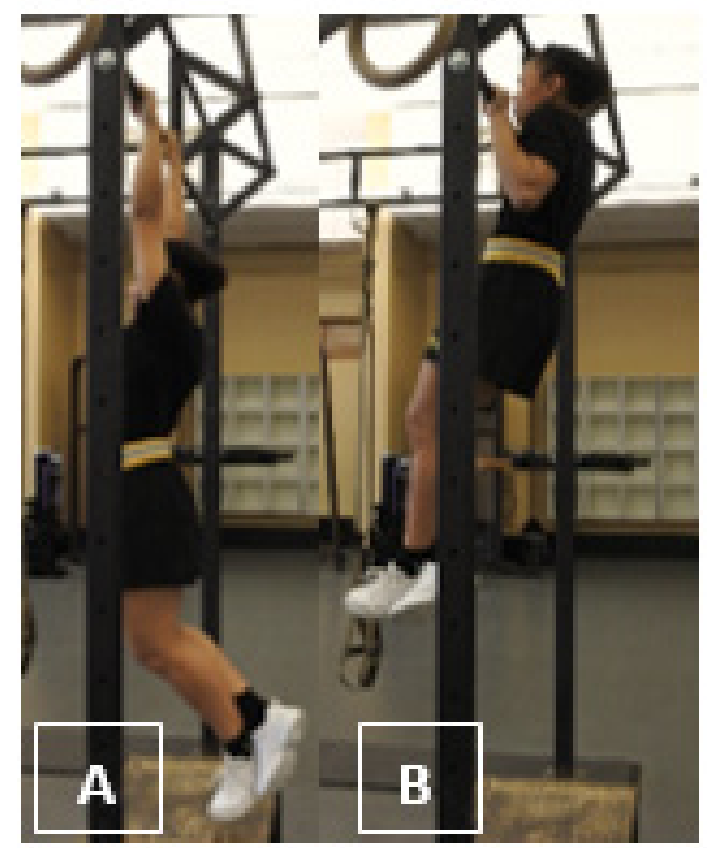

Figure 2: The body weight pull-up (PU) shown in the starting position (A) and up position (B).

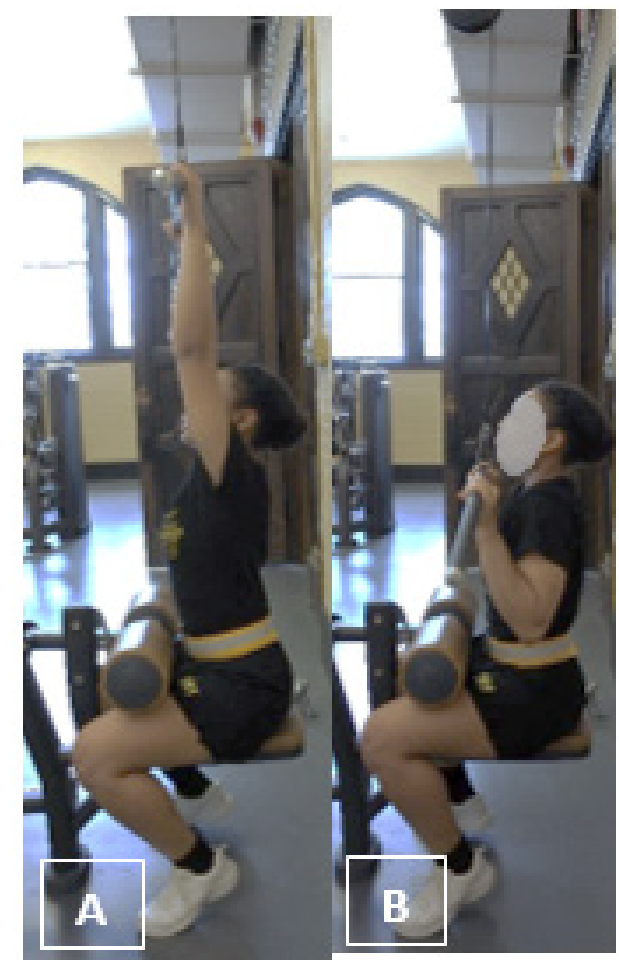

Figure 3: The seated lat-pulldown (s-LP) shown in the starting position (A) and up position (B). 


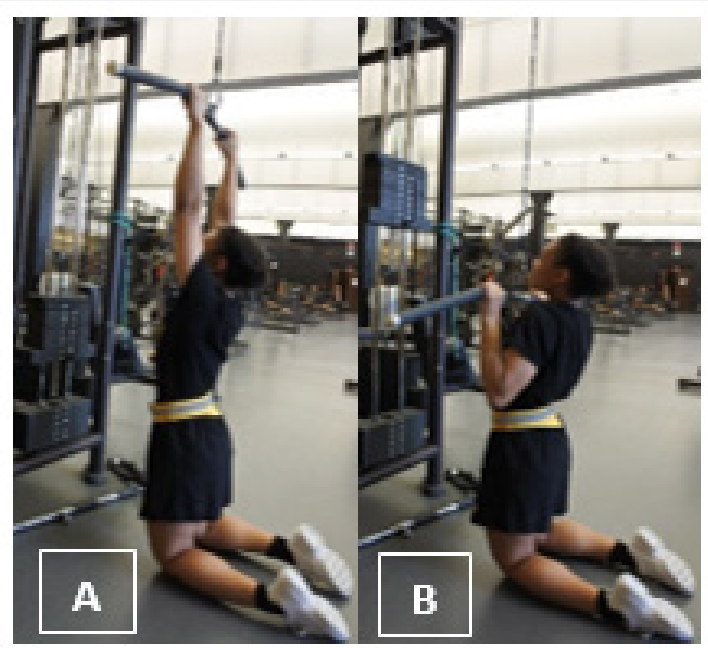

Figure 4: The kneeling lat-pulldown (k-LP) shown in the starting position (A) and up position (B).



Figure 5: The assisted pull-up (aPU) shown in the starting position (A) and up position (B).

\section{Data Analysis}

For analysis, participants' data were grouped based off of the reported max pull-up number (i.e. under 10 reps (u10) and 10 and over reps (10+)). Raw EMG data were sampled at $2000 \mathrm{~Hz}$ using a Butterworth filter with corner frequencies of $20-450 \mathrm{~Hz}$ and a slope of $12 \mathrm{db} /$ oct. Data was then smoothed using the root mean square (RMS) Trigno software calculation (Delsys EMG Works Analysis software). The peak EMG magnitudes were recorded for the 5-second MVIC bout for each muscle and the $2^{\text {nd }}, 3^{\text {rd }}$ and $5^{\text {th }}$ repetitions of each exercise. The peak magnitudes were then averaged for the 3 repetitions. Mean peak magnitudes for each muscle are represented as percentages of MVIC (\%MVIC).

\section{Statistical Analysis}

Pearson correlation coefficients were used to determine the strength of the relationship of the activity in each of the 4 muscle groups of interest for the PU and each individual alternative pulling exercise (sLP, kLP and aPU) for the two groups. Coefficient of determination ( $\mathrm{r} 2$ ) was then used to determine the extent that the magnitude of activity in the respective alternative exercise explained the magnitude of activity in the PU. An alpha level of $\mathrm{p} \leq$ 0.05 was set for the level of significance for all statistical analyses. Sigma Stat 4.0 for Windows (Systat Software, Inc., San Jose, CA) was used to perform all statistical analyses.

\section{Results}

Of the four muscle groups tested, the greatest (normalized) magnitudes of muscle activity observed during the PU for the u10 group were: RA $(1.58 \pm 0.94)$, BI $(1.54 \pm 0.52)$, LAT $(1.20 \pm 0.67)$, and finally TRAP $(0.93 \pm 0.56)$ see Table 2 . The k-LP was the only alternative pulling exercise observed to have the same activation pattern. In contrast, the $10+$ group had the greatest magnitude of 
action in the BI $(1.22 \pm 0.45)$ followed by the RA $(1.02 \pm 0.75)$, LAT $(0.96 \pm 0.42)$ and finally the TRAP $(0.74 \pm 0.65)$. None of the alternative pulling exercises were observed to have the same magnitude of activation pattern as the pull-up. For the u10 Reps group, Pearson Correlation Coefficient indicated very strong relationships between the magnitude of RA activity in the PU and both the s-LP and k-LP ( $r=0.90, p<0.001)$, as well as between BI activity in the PU and both the s-LP and k-LP ( $\mathrm{r}=0.91$ and 0.89 , respectively, $\mathrm{p}<0.001$ ) and the LAT activity in the PU and k-LP ( $r$ $=0.90, \mathrm{p}<0.001$ ) see Table 2 . Moderate to low relationships in the TRAP were observed between the PU and all three alternative exercises ( $\mathrm{r}=0.35$ to $0.69, \mathrm{p}=0.01$ to 0.221 ). The greatest shared variance of the muscles and exercises of interest were observed in the RA between the PU and k-LP, and PU and s-LP (r2 = 0.81), $\mathrm{BI}$ between the PU and s-LP, and PU and k-LP (r2 = 0.83 and 0.79, respectively), and the LAT between the PU and $\mathrm{k}-\mathrm{LP}(\mathrm{r} 2=0.81)$. The correlational analysis for the $10+$ Reps group revealed very strong relationships between the TRAP activity for the PU and aPU $(\mathrm{r}=0.95, \mathrm{p}<0.001)$ as well as the BI activity for the PU and k-LP ( $\mathrm{r}$ $=0.85, \mathrm{p}<0.001)$. Moderate to low relationships were observed for all other magnitudes and exercises. The greatest shared variance was observed in the TRAP between the PU and aPU (r2 = 0.90) see Table 2 .

Table 2: Statistical analyses for each muscle during each exercise.

\begin{tabular}{|c|c|c|c|c|c|c|c|}
\hline \multirow{2}{*}{\multicolumn{2}{|c|}{$\mathbf{r}$}} & \multicolumn{3}{|c|}{ U10 Reps Group } & \multicolumn{3}{|c|}{ 1+0 Reps Group } \\
\hline & & $\mathbf{R}$ & p-value & & $\mathbf{R}$ & p-value & \\
\hline \multirow{4}{*}{ PU vs s-LP } & RA & 0.90 & 0.81 & $<0.001^{*}$ & 0.49 & 0.24 & $0.01^{*}$ \\
\hline & $\mathrm{BI}$ & 0.91 & 0.83 & $<0.001^{*}$ & 0.81 & 0.66 & $<0.001^{*}$ \\
\hline & LAT & 0.86 & 0.74 & $<0.001^{*}$ & 0.73 & 0.53 & $<0.001^{*}$ \\
\hline & TRAP & 0.35 & 0.12 & 0.22 & 0.86 & 0.74 & $<0.001^{*}$ \\
\hline \multirow{4}{*}{ PU vs k-LP } & RA & 0.90 & 0.81 & $<0.001^{*}$ & 0.57 & 0.32 & $<0.001^{*}$ \\
\hline & $\mathrm{BI}$ & 0.89 & 0.79 & $<0.001^{*}$ & 0.85 & 0.72 & $<0.001^{*}$ \\
\hline & LAT & 0.90 & 0.81 & $<0.001 *$ & 0.75 & 0.56 & $<0.001^{*}$ \\
\hline & TRAP & 0.69 & 0.48 & $0.01^{*}$ & 0.56 & 0.31 & $<0.001^{*}$ \\
\hline \multirow{4}{*}{ PU vs aPU } & RA & 0.58 & 0.34 & $0.03^{*}$ & 0.57 & 0.32 & $<0.001^{*}$ \\
\hline & BI & 0.83 & 0.69 & $<0.001^{*}$ & 0.76 & 0.58 & $<0.001^{*}$ \\
\hline & LAT & 0.08 & 0.01 & 0.80 & 0.60 & 0.36 & $<0.001^{*}$ \\
\hline & TRAP & 0.64 & 0.41 & $0.01^{*}$ & 0.95 & 0.90 & $<0.001^{*}$ \\
\hline
\end{tabular}

\section{Discussion and Conclusion}

This study sought to investigate whether muscle activation patterns are similar between the traditional body weight pullup and various alternative pulling exercises often used to train for improved performance in the pull-up. Of the four muscle groups assessed, the RA was the most active muscle group for all participants when performing the PU. High activation levels of the core musculature are not surprising as this muscle group helps to maintain a rigid body position throughout the PU. Previous research has supported this notion when assessing RA activity during various other movements that require core stabilization (e.g. supine v-up, prone suspension exercises, push-ups, squats, etc.) wherein, the RA are activated to a greater extent as the performance surface becomes less stable. Anderson and Behm [16], Cugliari and Boccia [17], Garg and Sharma [18], Snarr, Esco, Witte, Jenkins, \& Brannan [19], Snarr, Hallmark and Esco [20] Van den Tillaar and Saeterbakken [21]. The PU is performed while being suspended from a bar, and therefore requires greater stabilization of the core throughout the execution of the exercise, than would be required if performed from a solid base of support (e.g. seated lat pulldown).

Of the three alternative pulling exercises, only the k-LP replicated the order of activation pattern for the u10 Rep group (from greatest to least active); RA, BI, LAT, and TRAP. This indicates that as participants near their maximal effort during both the PU and alternative pulling exercise, only the k-LP activate these four muscle groups to relatively the same extent as that of the PU. This inference is further supported by the relatively high shared variance between the PU and k-LP for the RA muscle group. Additionally, when performing the k-LP, participants were pulling a load that was equal to $80 \%$ of their body weight. As a result, the participants were lifted off of the ground for a portion of the pulling (concentric) phase of the movement, similar to the suspension aspect of the pull-up. Unfortunately, there is a lack of research in this area, and therefore it is difficult to determine if the data presented in the current study is an accurate representation of a larger population. In contrast to the u10 Rep group, aside from the RA (most active for all exercises) none of the activation patterns for the alternative pulling exercises for the 10+ Reps group replicated that of the PU see Figure 6. As only 5 reps were performed by each participant, those that self-reported to be able to perform at least 10 reps would have performed at a submaximal level for each of these exercises. As such, it is likely that as the performance nears maximal effort, the activation patterns shift to accommodate the more challenging load. 


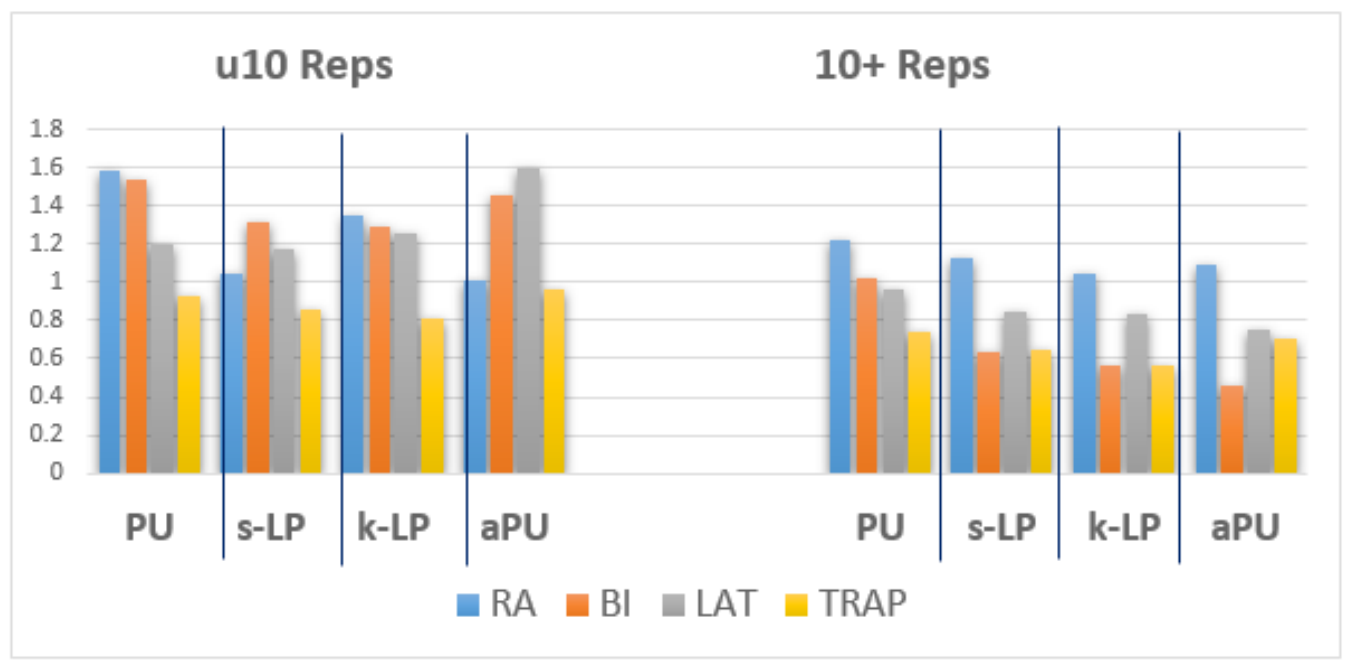

Figure 6: Magnitude of muscle activity for each exercise for the u10 Reps group (left) and 10+ Reps group (right) represented as \%MVIC. Note: $\mathrm{PU}=$ Pull-up; s-LP = Seated Lat-Pulldown; k-LP = Kneeling Lat-Pulldown; $\mathrm{aPU}=$ Assisted Pull-up; $\mathrm{RA}=$ Rectus Abdominis; $\mathrm{Bi}=$ Biceps Brachii; LAT = Latissimus Dorsi; TRAP = Trapezius.

The 3 alternative pulling exercises each had the second largest activation from the LATS, followed by the TRAP and BI. As the 3 alternative pulling exercises were each performed at $80 \%$ of BW, it is plausible that the activation pattern would shift more towards the BI if the load was set to $100 \%$ BW (i.e. to replicate the load of the pull-up). Unfortunately, the current study did not account for this and there is a paucity of previously published investigating both the muscle activation patterns as well as the comparison of alternative pulling exercises. Previous studies have focused primarily on EMG magnitudes when using different hand placements (e.g. narrow, wide, shoulder-width, etc.), hand grips (e.g. pronated, supinated, etc.) and movement techniques (e.g. behind the neck versus in front of the neck, PU with rings, kipping PU, etc.) for the PU and lat-pulldown exercises Andersen [15], Dickie [10], Lusk [11], Signorile [12], Snarr [8], Snarr [9], Sperandei [13], Youdas [22]. This made it difficult to compare current EMG data to that of previously published data, specifically with the k-LP variation. Additionally, while the current study assessed the 4 muscles thought to be prime movers and prime stabilizers in the PU (RA, LAT, BI and TRAP), previous research has focused more on the use of shoulder muscles (e.g. deltoid, teres major, serratus anterior, etc.) to help stabilize the highly mobile glenohumeral and scapulothoracic joints through such a challenging motion Andersen [15], Dickie [10], Signorile [12], Snarr [8], Snarr [9], Sperandei [13], Youdas [22].

However, the intention of the current study is to investigate the PU and alternative pulling exercises using a novel approach for future research to evolve from. Based solely off of muscle activation patterns, it appears that the k-LP is the most similar to the PU for near maximal efforts. For submaximal training purposes, it is unclear which of the alternative training exercises has the most similar activation pattern as the PU. It is plausible that a different alternative pulling exercise might be better related to the PU when training sub maximally. It is clear, however, that the RA should be emphasized in training programs as it was the most active muscle group assessed for the PU and k-LP (all participants), and s-LP and aPU for submaximal efforts. While additional research is greatly needed, based off initial muscle activity and shared variance with the PU, the k-LP (at $80 \%$ BW) is the best alternative exercise to the traditional PU compared to the s-LP and aPU. Current research by the authors of this article is aimed at investigating if muscle activation patterns change as participants reach temporary muscle failure in the PU (i.e. potential compensational strategies or increased muscle effectiveness); as well as establishing normative data for males and females when performing these four exercises.

\section{Acknowledgement}

The authors would like to thank all the participants for their time and effort during data collection.

\section{References}

1. Astrand P, Rodahl K, Dahl H, Stromme S (2003) Physical Training Textbook of Work Physiology; Physiological Bases of Exercise $4^{\text {th }}$ ed., Champaign, IL, Human Kinetics, USA pp. 346-347.

2. Bompa T (2009) Periodization Theory and Methodology of Training ( $5^{\text {th }}$ ed.). Champaign, IL, Human Kinetics, USA.

3. MacIntosh B, Gardiner P, McComas A (2006) Muscle Training Skeletal Muscle Form and Function $2^{\text {nd }}$ ed., Champaign, IL, Human Kinetics, USA pp. 298-300.

4. Baumgartner T, Gaunt S (2005) Construct Related Validity for the Baumgartner Modified Pull-up Test. Measurement in Physical Education and Exercise Science 9(1): 51-60.

5. Halet K, Mayhew J, Murphy C, Fanthorpe J (2009) Relationship of 1 repetition maximum lat-pull to pull-up and lat-pull repetitions in elite collegiate women swimmers. Journal of Strength and Conditioning Research 23(5): 1496-1502.

6. Johnson D, Lynch J, Nash K, Cygan J, Mayhew J (2009) Relationship of lat-pull repetitions and pull-ups to maximal lat-pull and pull-up strength in men and women. Journal of Strength and Conditioning Research 23(3): 1022-1028. 
7. Ronai P, Scibek E (2014) The Pull-up. Strength and Conditioning Journal 36(3): 88-90.

8. Snarr R, Casey J, Hallmark A, Esco M (2018) Electromyographical Comparison of a Traditional and Kipping Pull-up. Journal of Australian Strength and Conditioning 26(2): 28-34.

9. Snarr R, Hallmark A, Casey J, Esco M (2017) Electromyographical Comparison of a Traditional, Suspension Device, and Towel Pull-up. Journal of Human Kinetics 58: 5-13.

10. Dickie J, Faulkner J, Barnes M, Lark S (2017) Electromyographic analysis of muscle activation during pull-up variations. Journal of Electromyography and Kinesiology 32: 30-36.

11. Lusk S, Hale B, Russell D (2010) Grip width and forearm orientation effects on muscle activity during the lat pull-down. Journal of Strength and Conditioning Research 24(7): 1895-1900.

12. Signorile J, Zink A, Szwed S (2002) A Comparative Electromyographical Investigation of Muscle Utilization Patterns Using Various Hand Positions During the Lat Pull-down. Journal of Strength and Conditioning Research 16(4): 539-546.

13. Sperandei S, Barros M, Silveira Junior P, Oliveira C (2009) Electromyographic Analysis of Three Different Types of Lat Pull-down. Journal of Strength and Conditioning Research 23(7): 2033-2038.

14. Konrad P (2006) The ABC of EMG - A practical introduction to kinesiological electromyography (Vol. 1.4). Scottsddale, AZ: Noraxon USA, Inc.

15. Andersen V, Fimland M, Wiik E, Skoglund A, Saeterbakken A (2014) Effects of Grip Width on Muscle Strength and Activation in the lat Pull- down. Journal of Strength and Conditioning Research 28(4): 11351142.

16. Anderson K, Behm D (2005) Trunk Muscle Activity Increases with Unstable Squat Movements. Canadian Journal of Applied Physiology 30(1): 33-45.

17. Cugliari G, Boccia G (2017) Core Muscule Activation in Suspension Training Exercises. Journal of Human Kinetics, USA 56: 61-71.

18.Garg C, Sharma R (2013) Rectus Abdominis Muscle Activity on Different Surfaces. Physiotherapy and Occupational Therapy Journal 6(3): 109-115.

19. Snarr R, Esco M, Witte E, Jenkins C, Brannan R (2013) Electromyographic Activity of Rectus Abdominis during a Suspension Push-up Compared to Traditional Exercises. Journal of Exercise Physiology Online 16(3): $1-8$

20. Snarr R, Hallmark A, Esco M (2016) Core Muscle Activation During Traditional Abdominal Exercises: An Electromyographical Comparison. Journal of Sport and Human Performance 4(2): 1-7.

21. Van den Tillaar R, Saeterbakken A (2018) Comparison of Core Muscle Activation Between a Prone Bridge and 6-RM Back Squats. Journal of Human Kinetics 62: 43-53.

22. Youdas J, Amundson C, Cicero K, Hahn J, Harezlak D, et al. (2010) Surface Electromyographic Activation Patterns and Elbow Joint Motion During a Pull-up, Chin-up, or Perfect Pull-up (TM) Rotational Exercise. Journal of Strength and Conditioning Research 24(12): 3404-3414.

\section{Your next submission with Juniper Publishers will reach you the below assets}

- Quality Editorial service

- Swift Peer Review

- Reprints availability

- E-prints Service

- Manuscript Podcast for convenient understanding

- Global attainment for your research

- Manuscript accessibility in different formats ( Pdf, E-pub, Full Text, Audio)

- Unceasing customer service

Track the below URL for one-step submission https://juniperpublishers.com/online-submission.php 